\title{
Evaluación de metodologías para la construcción de mercados bursátiles artificiales.
}

\author{
Assessment of artificial stock market building strategies. \\ Leonardo Talero Sarmiento - Juan Benjamín Duarte Duarte \\ Katherine Julieth Sierra Suárez
}

COLOMBIA

Resumen: El objetivo principal del presente trabajo es evaluar las metodologías para la construcción de mercados bursátiles artificiales, determinando, primero, un modelo generador de precios para el mercado colombiano; segundo, simulando el modelo autómata conductual propuesto por Fan en 2009; y, tercero, aplicando un modelo racional propuesto mediante la adaptación de los Filtros de Alexander. Los modelos son contrastados mediante su consenso (volumen de compras, retenciones y ventas). Durante esta investigación se establece que el modelamiento de agentes racionales permite un comportamiento menos predictivo y un mayor impacto en el uso de la información, similar a la Hipótesis de Eficiencia del Mercado; además, los mercados bursátiles celulares conductuales y racionales no convergen; por ende, se plantea para futuras investigaciones la necesidad de construir modelos racional-conductuales con algoritmos de estimación de precios.

Palabras Claves: Autómatas celulares; decisiones racionales; finanzas conductuales; psicología de inversores; simulación.

Abstract: The primary objective of this paper is to show the methodology assessment to construct artificial stock markets, determining: first, a Colombian price generator model; second, modifying the cellular automaton model developed by Fan in 2009; third, building a rational automaton model, adapting the Alexander Filters methodology. The models are compared using their consensus (volume of Buyers, Holders, and Sellers). This research determines that rational agent-based modeling allows a less predictive behavior and a greater impact on the information use, similar to the Efficient Market Hypothesis; moreover, there is no convergence between rational and behavioral artificial stock markets. Therefore, we propose a rational-behavioral model abreast price estimation algorithm as a future research proposal.

Keywords: rational decisions; behavior finances; cellular automata; investor psychology; simulation.

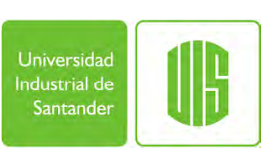

Leonardo Talero es Ingeniero Industrial de la Universidad Industrial de Santander, estudiante de Maestría en Ingeniería Industrial, de la Facultad de Ingenierías Físico-Mecánicas, Escuela de Estudios Industriales y Empresariales, Bucaramanga, Colombia.

Contacto: leonardo.talero@correo.uis.edu.co 


\section{Avaliação de metodologias para a construção de mercados artificiais}

Resumo: O principal objetivo deste estudo é avaliação de metodologias para a construção de mercados artificiais de ações: em primeiro lugar, um modelo de preços gerador para o mercado colombiano; segundo, simulando o modelo de comportamento autômato proposto por Fan em 2009; terceiro, um modelo racional proposto pela adaptação Filtros Alexander. Os modelos são contrastados pelo seu consenso (volume de o que comprar, manter ou vender). Durante esta investigação determinou-se que a modelagem de agentes racionais permite um comportamento menos previsível e um maior impacto sob o uso da informação semelhante de hipótese da eficiência do mercado; também que os mercados de ações celular comportamentais e racionais não convergem, desta forma nós propomos construir um modelo comportamental-racionais acompanhados de algoritmos de estimação preços como futura proposta de investigação.

Palavras-chave: autômatos celulares; decisões racionais; finanças comportamentais; psicologia dos investidores; simulação.

\section{Introducción}

egún Francisco Formariz, "la Bolsa es un mercado. Como en todo mercado, se negocia una serie de productos y se ponen en contacto compradores y vendedores" (Blásquez, 2000:1), por tanto, es un punto de encuentro en el que el equilibrio entre oferentes y demandantes permite el crecimiento de la economía, puesto que le ofrece a los inversores la posibilidad de transformar en el corto o inmediato plazo los valores mobiliarios en dinero $y$, a la par, funge como herramienta de financiación empresarial.

Dicho espacio de negociación es de vital importancia para las naciones, ya que refleja el comportamiento, estado y esperanza de una economía. Ese estado, a su vez, es el resultado de las propuestas de mejora, crecimiento, generación de valor empresarial y de cómo las personas reconocen dicha gerencia; es decir, toda propuesta de integración en la cadena de valor, expansión de mercados, venta de acciones y demás posibles decisiones

\footnotetext{
Juan Duarte es Ingeniero industrial, Doctor en Finanzas de Empresas, profesor titular de la Universidad Industrial de Santander, Facultad de Ingenierías FísicoMecánicas, Escuela de Estudios Industriales y Empresariales, Bucaramanga, Colombia.

Contacto: jduarte@uis.edu.co.

Katherine Sierra es Ingeniera industrial, Magíster en Ingeniería Industrial, profesora de la Universidad Industrial de Santander, Facultad Ingenierías FísicoMecánicas, Escuela de Estudios Industriales y Empresariales, Bucaramanga, Colombia.

Contacto: katjulss@gmail.com
}

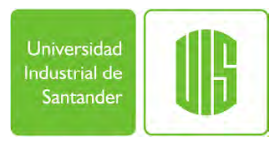


de la alta gerencia es reconocida por los stakeholders', quienes adoptan ciertas posiciones como comprar, retener o vender una acción, cambiando el valor percibido de la empresa.

Comprender la dinámica de la Bolsa implica conocer la mecánica de negociación de activos financieros y el comportamiento de los mismos. Uno de los primeros trabajos en analizar y condensar la naturaleza de las acciones (reflejado en los precios) fue el realizado por Gibson (1889), quien tomaba a modo base la libre información y, por tanto, la igualdad de oportunidades al momento de negociar como el motor de la oferta y demanda. Con el tiempo, diversos investigadores continuaron analizando los mercados bursátiles y planteando hipótesis que buscaban entender la naturaleza de la Bolsa y los activos cotizados en ella. Entre las hipótesis se destacan la Eficiencia del Mercado (Fama, 1970), la cual plantea que los inversores toman decisiones basados en la razón y, por ende, los precios de los activos cotizados son impredecibles, ya que reflejan toda la información disponible; la Hipótesis de Mercado Fractal (Mandelbrot, 1972), que expone que los precios de los activos tienen cierto nivel de memoria relacionada con los horizontes temporales de los inversionistas y que la predicción de su ciclo es posible a partir del estudio fractal de las series; la Hipótesis de Mercado Conductual (Shiller, 2003), (Shiller, 2006), que manifiesta que los inversores no siempre son racionales $\mathrm{y}$, en consecuencia, las anomalías evidenciadas mediante estudios empíricos como el efecto manada, efecto tamaño y demás son derivados de las decisiones no racionales de los inversores. Y la hipótesis del Mercado Adaptativo (Lo, 2005), la cual vincula el comportamiento eficiente y conductual de los inversores con principios de evolución como competencia, adaptación y selección natural.

El presente trabajo busca recopilar metodologías para la estimación del precio de las acciones y ver si estas se pueden integrar al modelo de bursátil artificial propuesto por Fan (Fan, Ying, Wang, \& Wei, 2009) y compararlas a partir del concepto de consenso. La estructura a seguir abarca un recorrido por el primer capítulo en el que se exponen antecedentes conceptuales, seguida del segundo capítulo en el que se desarrolla la metodología, la cual, en una primera instancia, trata de cuatro modelos para la generación de precios y, en una segunda, expone la estructura de interacción del mercado bursátil virtual planteado por Fan y el propuesto durante esta investigación. En el tercer capítulo, denominado simulación y análisis, se realizan las comparaciones entre los modelos a partir de las

1 Todos los interesados de una empresa suelen ser los accionistas o agentes dispuestos a interactuar en sus activos financieros. 
posiciones de los agentes. El cuarto capítulo se refiere a las conclusiones de la investigación y finalmente, el quinto, a la bibliografía empleada.

\section{Antecedentes}

La economía financiera se ha enriquecido a lo largo de su haber por teorías que buscan explicar el comportamiento de los precios de diversos activos económicos. En su tesis doctoral Bachelier (1900) propone que el precio sigue una distribución de probabilidad normal, abriendo las puertas del estudio de series financieras desde la perspectiva probabilística. Diversos autores indagan la razón por la cual dichos precios fluctúan ante la misma información (Keynes, 1923), (Sidney, 1961) (Fama, 1965), postulando, en consecuencia, la Hipótesis de Eficiencia del Mercado (HEM) (Fama, 1970), la cual expone que, debido a la sinergia de la Amplitud, Transparencia, Libertad, Profundidad y Flexibilidad de los mercados, el precio de los activos financieros es impredecible y este representa toda la información disponible en el momento.

A la par del desarrollo computacional, análisis de datos y modelamiento de funciones comenzaron a tomar fuerza la construcción de algoritmos que representaran la realidad. Ashby (1987) recomendaba que para el buen funcionamiento de los programas informáticos era necesario separar el modelador de la máquina a través del comportamiento aleatorio de sus variables independientes, evitando así sesgar las configuraciones. Siguiendo la propuesta del estudio de números pseudoaleatorios generados artificialmente y el análisis de los mismos mediante técnicas estadísticas, Peters consigna en un libro el estudio de las series financieras a partir del reescalamiento de sus datos bajo el supuesto de independencia y aleatoriedad, generando aportes en la teoría del caos en el mercado bursátil y el análisis de las series mediante su tendencia fractal (Peters, 1994), acorde a lo propuesto por Mandelbrot en su Hipótesis de Mercado Fractal (HMF).

En aras de encontrar una metodología que integrara el análisis de series de tiempo junto con el modelamiento aleatorio de activos financieros, Lebaron propone la construcción de un mercado bursátil artificial basado en un pequeño número de comportamientos determinados, el cual llamó Modelo de pocos tipos. (LeBaron, Arthur \& Palmer, 1999). Por otra parte, Levy y otros investigadores abordaron el concepto de simulación del mercado a partir de la interacción entre agentes, quienes buscaban maximizar su utilidad o riqueza esperada (Levy, Levy, \& Solomon, 2000).

Las diversas hipótesis financieras, en conjunto con las propuestas de integración y análisis de datos, brindan una herramienta para la validación de supuestos porque, como lo expone Olmeda, "ciertas series 
aparentemente aleatorias, como las bursátiles, pudieran estar generadas por procesos deterministas, de manera que una vez determinado el proceso generador fuera posible emplearlo para realizar predicciones" (Blásquez, 2000:233). Dichos estudios desde la perspectiva computacional se ven consignados en los aportes de Fan (Fan et al., 2009), Milan (Lovric, 2011) y Mozafari (Mozafari \& Alizadeh, 2013).

Bajo ese marco de importancia, Duarte y Sierra realizan estudios cobre el caos y eficiencia en mercados bursátiles latinoamericanos (Sierra Suárez, Duarte Duarte, \& Mascareñas Pérez-Iñigo, 2013), (Duarte Duarte \& Mascareñas Pérez-Iñigo, 2014b), los cuales fungirán como punto de partida para la comprobación de la teoría de finanzas conductuales en el mercado bursátil de Colombia, desarrollada por Talero (Talero Sarmiento, 2015).

\section{Metodología}

\subsection{Simulación para la generación de precios}

Los agentes en un mercado bursátil están dispuestos a sacrificar parte de su capital con el fin de invertirlo en activos financieros, buscando así obtener el retorno de la inversión sumada a unas ganancias. Dicho nivel de sacrificio genera el precio de un activo en el mercado (Lovric, 2011), en cada momento las variables que permiten adoptar una posición u otra cambian, la interacción de esos factores genera un precio y, por ende, este es dinámico e impredecible. La rentabilidad es, entonces, un análisis del cambio, tendencia y volatilidad del nivel de sacrificio de los inversores.

Estimar el precio de un activo determinado, en un tiempo específico y dentro de un mercado bursátil virtual se puede lograr mediante diversas técnicas. En el marco de esta investigación se enfatizan cuatro metodologías recopiladas por Milan, listadas a continuación:

Equilibrio temporal del mercado: Es un mecanismo donde los precios son determinados por la demanda total de los agentes, y cuando esta se iguala al número total de acciones en el mismo periodo, se genera una demanda para cada agente, acompañado de un precio estratégico y este va cambiando hasta encontrar el equilibrio.

Función del impacto del precio: Se estructura un proceso de ajuste en el que el precio futuro es determinado por el precio pasado y una orden de pedido (la diferencia entre la demanda y la oferta), adaptada por un parámetro $\alpha$. El exceso de demanda incrementa el precio, mientras que el exceso de oferta lo disminuye. La ventaja de esta metodología es la velocidad computacional; sin embargo, la estimación es sensible al parámetro de liquidez $\alpha$. 
Ecuación 1 Función del impacto del precio

$$
P_{t+1}=P_{t}+\propto *\left(D *\left(P_{t}\right)-S *\left(P_{t}\right)\right)
$$

Cartera de pedidos: Es un mecanismo de asignación de precios en el que la cartera de pedido es simulada, los agentes se entretejen mediante un proceso, negociando y generando el valor. Es una metodología más realista porque tiene en consideración diversos mecanismos de negociación y contrasta con el modelo basado en el equilibrio, puesto que, en ese, la negociación explícita no es modelada.

Emparejamiento: Se genera una cantidad aleatoria de agentes y estos, de forma probabilística, se encuentran y negocian unos con otros. Es un mecanismo que permite modelar situaciones donde los mercados de valores no están bien definidos o establecidos; luego, entonces, no existen instituciones que ayuden a los vendedores a disminuir el ajuste aleatorio del precio.

Teniendo en cuenta que el mercado bursátil colombiano presenta un grado de madurez en el periodo comprendido entre 2002 y 2012 (Duarte Duarte, Mascareñas Pérez-Iñigo, \& Sierra Suárez, 2014), al seguir una Caminata Aleatoria Tipo I ${ }^{2}$ se descarta aplicar la metodología de Emparejamiento, puesto que es más útil cuando los mercados bursátiles están en desarrollo.

Por otra parte, como explica Sierra, el mercado bursátil colombiano presenta un comportamiento entre caótico y aleatorio:

Los periodos que muestran indicio de comportamiento caótico coinciden con los periodos con tendencia al alza, mientras que el aleatorio coincide con el periodo con tendencia a la baja. Esto podría explicarse por la hipótesis de mercado fractal de Peters (Peters, 1994), la cual postula que los inversores negocian con horizontes de largo plazo cuando el mercado es rentable, produciendo un equilibrio que hace posible realizar predicciones durante este periodo... (Sierra Suárez et al., 2013)

Por tanto, para esta investigación se recomienda modelar la metodología de Equilibrio temporal del mercado. Una vez ejecutado el pseudocódigo expuesto por Milan (Lovric, 2011:75), se analiza el comportamiento del precio durante 400 iteraciones mediante un diagrama de frecuencias de sus rentabilidades (ver ilustración 1). El comportamiento probabilístico de dicha función es similar a los estimados por Duarte (Duarte Duarte et 
al., 2014), con la diferencia de presentar una tendencia más leptocúrtica, similar a una función tipo Laplace, indicando que en el mercado colombiano existe una mayor variabilidad, la cual no es representada por agentes propuestos por Milan.

Gráfico 1. Ajuste para Rentabilidad Precio Estimado.

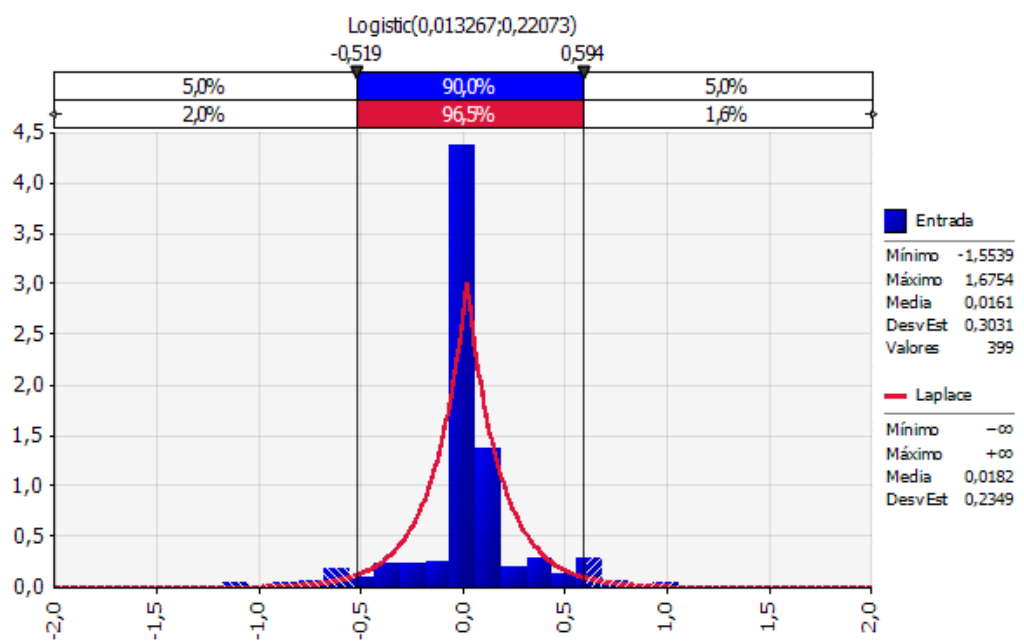

Fuente: Los autores a partir de @Risk

\subsection{Simulación del mercado bursátil virtual}

El mercado bursátil artificial planteado por Fan en 2009 no se enfoca en la generación de precios y su relación directa con las diversas hipótesis anteriormente planteadas (estudiadas a través del análisis de rentabilidades); en vez de ello, estudia la complejidad a partir de las posiciones adoptadas por los agentes: comprar, retener y vender. Dichas posiciones se asemejan al comportamiento bursátil al relacionar las posturas de los agentes con la dinámica entre oferta-demanda de activos financieros y su impacto en el precio de los mismos. La comparación se realiza mediante la estimación del coeficiente de Hurst y su longitud de ciclo, con el fin de entender si existe un nivel de memoria en dichas series y si estas representan un sistema complejo o caótico.

Conociendo la interpretación de los datos, desdeestaperspectivase puede tomar el exponente de Hurst como índice de caracterización de complejidad. En esta lógica, un exponente de Hurst elevado indica la presencia de una complejidad baja-correlación positivamientras que un exponente de Hurst bajo indica la presencia de una complejidad alta -correlación negativa- como cita Quezada en su tesis doctoral (Quezada Len, 2007:187). 
Según Fan, la predilección o preferencia en el momento de invertir está definida como una emulación del comportamiento y es propia de cada agente. $P(i, j)$ determina la probabilidad de imitación de un inversor en el sitio, $(i, j)$ significa que ese estado cambiará con probabilidad de $P(i, j)$ del estado actual al estado de la vecindad, el cual es definido como posición que la mayoría de sus vecinos adoptaron en el instante t.

La probabilidad puede ser nula o completa; luego, entonces, $P(i, j) \in[0$ ,1]. Una probabilidad $P(i . j) \in[0,0.5)$ de inversión indica una preferencia por antiimitar, la cual se hace más fuerte en cuanto $\mathrm{P}(\mathrm{i}, \mathrm{j})$ se acerca a cero. Cuando la probabilidad $P(i, j) \in(0.5,1]$ su preferencia en inversión está ligada con la imitación del comportamiento de sus vecinos y se hace más fuerte entre más cercano sea su valor a 1 . En dado caso de presentar una $P(i, j)=0.5$, la preferencia de la inversión se define por la no imitación del comportamiento de la vecindad.

En el estudio realizado por Fan en 2009, por simplicidad consideraron una situación especial en la que la preferencia a la hora de invertir era la misma, por tanto $P(i, j)=P$ para todos los agentes en el mercado bursátil. En el mercado real, el comportamiento de los inversores posee una tendencia mayor o menor. En muchos casos, la probabilidad es a imitar $P(i, j) \geq 0.5$, lo cual es consistente con el principio de mayoría y se relaciona estrechamente con el efecto manada en las finanzas conductuales (Duarte Duarte, Garcés Carreño \& Sierra Suárez, 2016), por ende, para esta investigación se trabajará con un $P(i, j)$ individual y no global.

Porotra parte, se puede definir un macrofactorque representeel coeficiente de compra o venta $M f \in[0,1]$, este se relaciona con el comportamiento de la economía e incrementa la probabilidad de que el inversor compre, retenga o venda una acción. Cuando el macrofactor se ve afectado por una información positiva, las probabilidades de comprar aumentan $y$, por tanto, la cantidad de inversores adoptando dicha posición. Cuando la macroinformación o la información del macrofactor es negativa, las probabilidades de venta de los inversores van a incrementarse; el macrofactor es un coeficiente ajustado de transferencia de probabilidad que se ve moldeado por la información macro del entorno bursátil.

Durante la simulación y para cada iteración, la postura ${ }^{3}$ que la mayoría de los vecinos de la posición $(i, j)$ adopten influye en la compra, retención o venta de una acción, tal como se muestra en la Tabla 1; la vecindad matricial se enlista a continuación: 
Ecuación 2 Estado de la vecindad

$$
S(i, j) L(t)=\left\{\begin{array}{ccc}
S(i-1, j-1)(t) & S(i-1, j)(t) & S(i-1, j+1)(t) \\
S(i, j-1)(t) & S(i, j+1)(t) \\
S(i+1, j-1)(t) & S(i+1, j)(t) & S(i+1, j+1)(t)
\end{array}\right\}
$$

Donde $S(i, j)$ representa la ecuación de vecindad para cada momento $t$ y es afectada por la posición adoptada por los vecinos al comienzo de cada iteración.

Tabla 1. Matriz de transferencia de posiciones para agentes conductuales.

\begin{tabular}{|c|c|c|c|c|}
\hline $\begin{array}{c}\text { Macro } \\
\text { factores }\end{array}$ & $\begin{array}{l}\text { Posición } \\
\text { vecindad }\end{array}$ & \multicolumn{3}{|c|}{ Transferencia de Probabilidades } \\
\hline & & Comprar & Retener & Vender \\
\hline \multirow{3}{*}{$\begin{array}{c}\text { Información } \\
\text { positiva }\end{array}$} & Comprar & $(P+M f)$ & $(1-P-M f) * 0,5$ & $(1-P-M f) * 0,5$ \\
\hline & Retener & $(1-P)^{*}\left(0,5+0,5^{*} M f\right)$ & $P$ & $(1-P)^{*}\left(0,5-0,5^{*} M f\right)$ \\
\hline & Vender & $(1-P)^{*}\left(0,5+0,5^{*} M f\right)$ & $(1-P)^{*}\left(0,5-0,5^{*} \mathrm{Mf}\right)$ & $P$ \\
\hline \multirow{3}{*}{$\begin{array}{c}\text { Información } \\
\text { negativa }\end{array}$} & Comprar & $P$ & $(1-P)^{*}\left(0,5-0,5^{*} \mathrm{Mf}\right)$ & $(1-\mathrm{P})^{*}\left(0,5+0,5^{*} \mathrm{Mf}\right)$ \\
\hline & Retener & $(1-P)^{*}\left(0,5-0,5^{*} \mathrm{Mf}\right)$ & $P$ & $(1-\mathrm{P})^{*}\left(0,5+0,5^{*} \mathrm{Mf}\right)$ \\
\hline & Vender & $(1-P-M f)^{*} 0,5$ & $(1-P-M f) * 0,5$ & $(\mathrm{P}+\mathrm{Mf})$ \\
\hline
\end{tabular}

Fuente: Adaptado de la tabla propuesta por Fan (Fan et al., 2009) The effect of investor psychology on the complexity of stock market: An analysis based on cellular automaton model.

$P \quad=$ Probabilidad de transferencia en el mercado

$M f$ = Macrofactor económico del mercado

Para la construcción del modelo algunos valores se van a restringir al conjunto entre 0 y 1 , de manera tal que valores inferiores o superiores serán descartados. De la misma forma la suma de los valores para las tres probabilidades de transferencia para cada agente en el Autómata Celular deberá ser igual a 1 .

Donde:

$$
P(B)+P(H)+P(S)=1
$$

$P(B)$ es la probabilidad de compra de un agente, $P(H)$ es la probabilidad de retención de un agente y $P(S)$ la probabilidad de venta de un agente.

Teniendo en cuenta que el modelo planteado por Fan en 2009 se basa solamente en las posiciones adoptadas por los agentes mediante la influencia de su entorno y sesga el análisis de hipótesis como la del mercado eficiente expuesta por Fama en 1970, durante esta investigación 
se propone generar modelos autómatas celulares con un enfoque racional que servirán como base para las futuras comprobaciones y contrastes de las hipótesis mediante la simulación.

Para la construcción del modelo se debe definir una regla de evolución que simule una acción racional y no se limite a simplemente imitar. Para este caso se propuso una variante de los filtros de Alexander, técnica que según Duarte no aplica rigurosa inferencia estadística, mas tiene aceptación porque genera una dinámica a través de la gestión activa de series financieras (Duarte \& Mascareñas, 2014:368). “Consiste en invertir y desinvertir durante un determinado tiempo sobre la base de la regla de comprar el activo cuando su precio se incrementa $x \%$ y venderlo cuando su precio cae $x \% "$; no obstante, como el modelo propuesto carece de un generador de precios que siga la metodología de Equilibrio temporal del mercado, el filtro se comparará con un incremento o decremento porcentual $z \%$ en la posible compra.

Ecuación 3 Variación porcentual

$$
z \%=\left\|\frac{\# \text { Agentes comprando }}{\# \text { Agentes comprando }+\# \text { Agentes vendiendo }}-0.5\right\|
$$

Como el mercado absorbe toda la información disponible, se define un macrofactor económico o el estado general de la economía como una variable independiente y aleatoria que sigue una distribución uniforme continua comprendida en el intervalo $[0,1]$.

Este $M f$ funciona como un símil, ya que no representa el comportamiento económico global de una región -como un macrofactor real-, pero sirve para analizar la tendencia probable al cambio. Como el $M f$ es estimado durante cada iteración, este debe recalcularse y es independiente en el tiempo; además, como la información disponible afecta la posición final de los agentes, cuando $M f \geq 0.5$ la economía se considera al alza.

La matriz general de transferencia de posiciones, basada en el estado de la economía y el filtro de decisión, se evidencia en la Tabla 2, donde $f \%$ simboliza el valor del filtro de Alexander. Cada agente analiza la intención de compra, retención o venta del mercado completo como referencia del comportamiento de la economía. Se plantean tres escenarios base: filtro exigente en $5 \%$; máximo de permisibilidad $1 \%$; valor medio de $3 \%$.

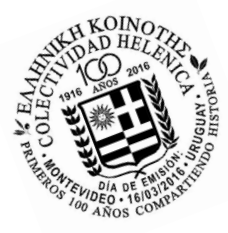


Tabla 2. Matriz de transferencia de posiciones para una gente racional.

\begin{tabular}{|c|c|c|c|c|}
\hline $\begin{array}{c}\text { Macro } \\
\text { factores }\end{array}$ & $\begin{array}{c}\text { Posición } \\
\text { vecindad }\end{array}$ & \multicolumn{3}{|c|}{ Transferencia de Posiciones } \\
\hline & & Comprar & Retener & Vender \\
\hline $\begin{array}{c}\text { Información } \\
\text { positiva }\end{array}$ & $\begin{array}{c}\text { Retener } \\
\text { Vender }\end{array}$ & $\mathrm{z} \%>\mathrm{f} \%$ & $\mathrm{z} \%=\mathrm{f} \%$ & $\mathrm{z} \%<\mathrm{f} \%$ \\
\hline \multirow{2}{*}{$\begin{array}{c}\text { Información } \\
\text { negativa }\end{array}$} & Retener & $\mathrm{z} \%<\mathrm{f} \%$ & $\mathrm{z} \%>\mathrm{f} \%$ & $\mathrm{z} \% \leq \mathrm{f} \%$ \\
& Vender & 0 & $\mathrm{z} \%>\mathrm{f} \%$ & $\mathrm{z} \% \leq \mathrm{f} \%$ \\
\hline
\end{tabular}

Fuente: Los autores

\section{Simulación y análisis}

El modelo autómata celular o Cellular Automata Model es programado en MATLAB y acompañado de un entorno gráfico o Graphical user Interface. Durante la simulación de los escenarios cada agente en la posición $(i, j)$ inicia con una postura: comprar, retener o vender (representada mediante un color) y es distribuida aleatoria y uniformemente en un espacio de 50*50 celdas (ver ilustración 2), de tal manera que existe una cantidad muy similar de agentes comprando, reteniendo y vendiendo.

Para cada inicio de periodo o iteración se vuelven a distribuir los agentes, quienes poseen una probabilidad de cambio $P(i, j)$ individual; esto implica que, para todas las iteraciones, un inversor en la posición $(i, j)$ observará el comportamiento de su entorno y teniendo en cuenta su capacidad de imitar o no cambiará de posición. La simulación se replica durante 100 veces, según lo propuesto en la investigación de Fan. La tendencia global de las posiciones o consenso indicará el efecto que tiene la manera de obtener la información y cómo esta favorece comprar, retener o vender una acción.

Tabla 3. Representación gráfica de las posiciones de los agentes en la vecindad.

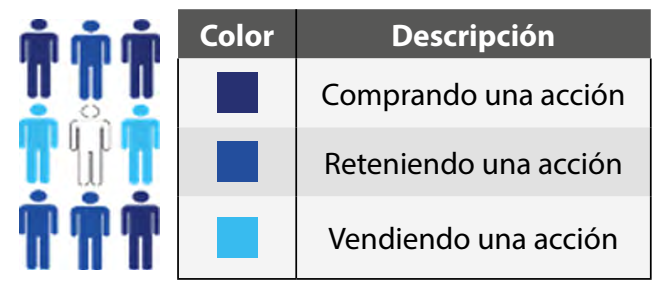

Fuente: Los autores 
Gráfico 2. Distribución de acciones

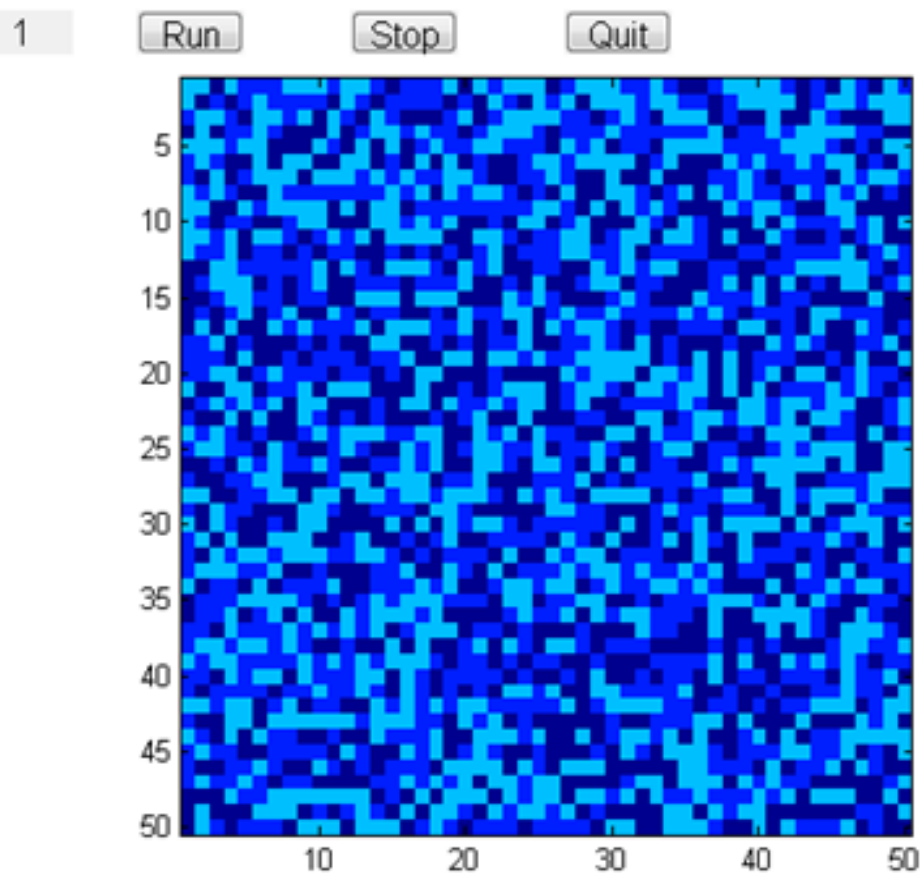

Fuente: Evaluación de las metodologías para la construcción de mercados artificiales. Talero (2015)

\subsection{Simulación para agentes conductuales}

Al simular el modelo autómata celular con probabilidad de imitación $P(i, j)$ individual y no global como el desarrollado por Fan, se observa una tendencia a comprar (ver ilustración 3). Esto implica que en un mercado ausente de racionalidad se presenta la anomalía denominada Efecto rebaño que impulsa la demanda u oferta, generando la volatilidad de activos financieros. Dicho comportamiento no suele ser el evidenciado en los mercados maduros como el de Estados Unidos ni se asemeja a aquellos que se están estabilizando como el de Colombia.

El consenso expuesto en un modelo virtual conductual con probabilidad de cambio individual no parece representar el comportamiento real de la bolsa colombiana y, en extensión, el de los mercados en madurez.

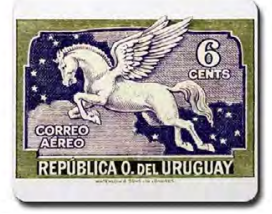


Gráfico 3. Consenso de agentes conductuales.

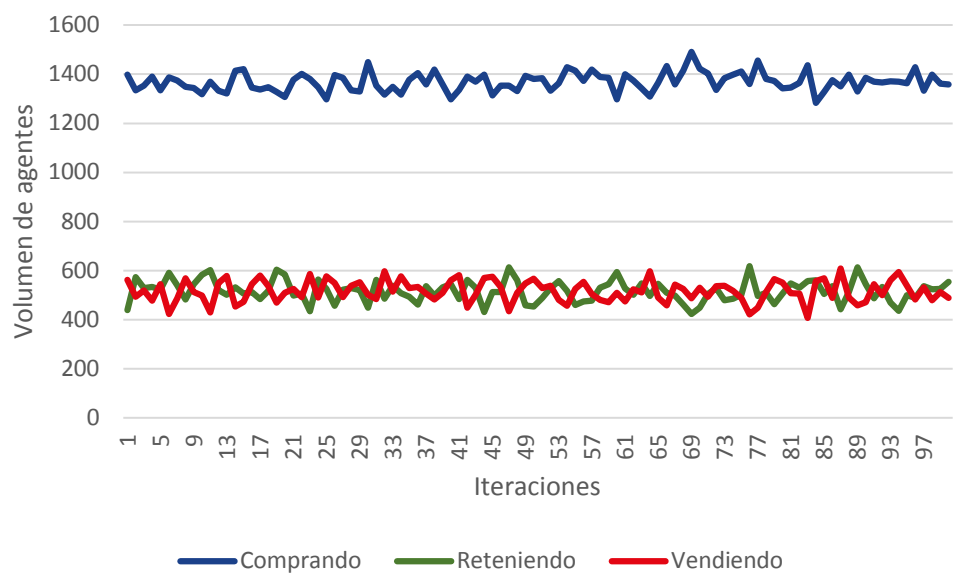

Fuente: Los autores

\subsection{Simulación para agentes racionales}

Al anular la probabilidad de imitación, permitiendo que el cambio de posición se deba a un ejercicio racional y modelando la tendencia de la economía como variables independientes, se evidencia una correlación positiva en las posiciones adoptadas durante la simulación (ver tabla 4), indicando que no existe preferencia por ninguna posición y el resultado final dependerá de la información disponible y el equilibrio entre la oferta y la demanda.

Al cambiar el nivel del filtro desde uno permisible (ilustración 4) hasta el exigente (ilustración 6), el consenso expuesto en un modelo virtual racional no presenta diferencia notoria; es decir, bajo el marco de un mercado eficiente es indiferente el nivel de exigencia que se tiene con la información y el consenso estimado o tendencia es estadísticamente la misma. Dicho comportamiento difiere con el real en diversas economías, puesto que la mayoría de las investigaciones en el área de finanzas conductuales rechaza la hipótesis de eficiencia del mercado (Duarte Duarte \& Mascareñas Pérez-Iñigo, 2014a), indicando que el modelo bursátil virtual debe tener componentes racionales y conductuales para asemejar la dinámica real de la Bolsa.

Tabla 4. Matriz de correlaciones de posiciones para un agente racional.

\begin{tabular}{|l|c|c|c|}
\hline & COMPRAR & RETENER & VENDER \\
\hline COMPRAR & 1 & & \\
RETENER & 0,9995834 & 1 & \\
VENDER & 0,99958223 & 0,99859734 & 1 \\
\hline
\end{tabular}

Fuente: Los autores 
Gráfico 4. Consenso de agentes racionales con máxima permisibilidad

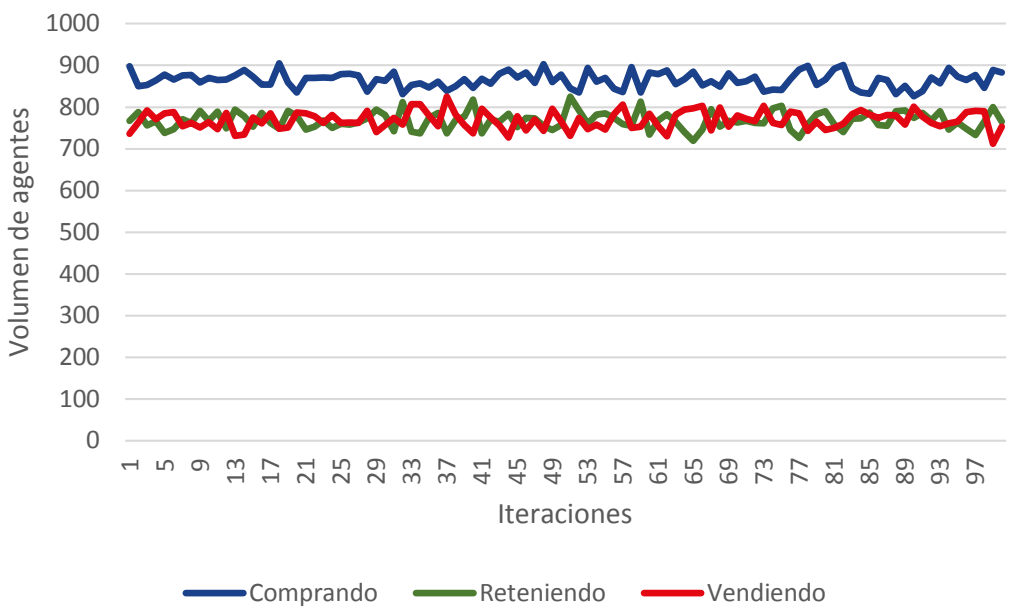

Fuente: Los autores

Gráfico 5. Consenso de agentes racionales con valor medio.

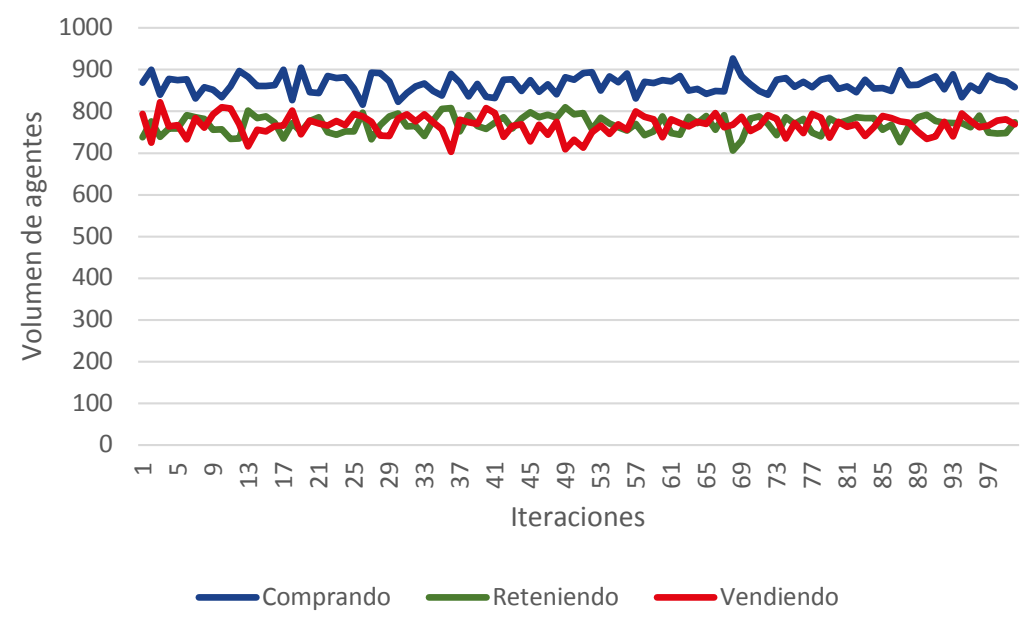

Fuente: Los autores

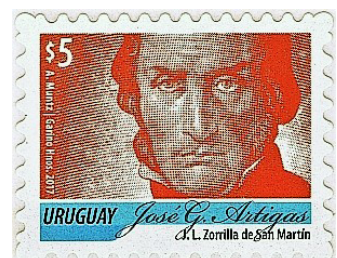


Gráfico 6. Consenso de agentes racionales con filtro exigente

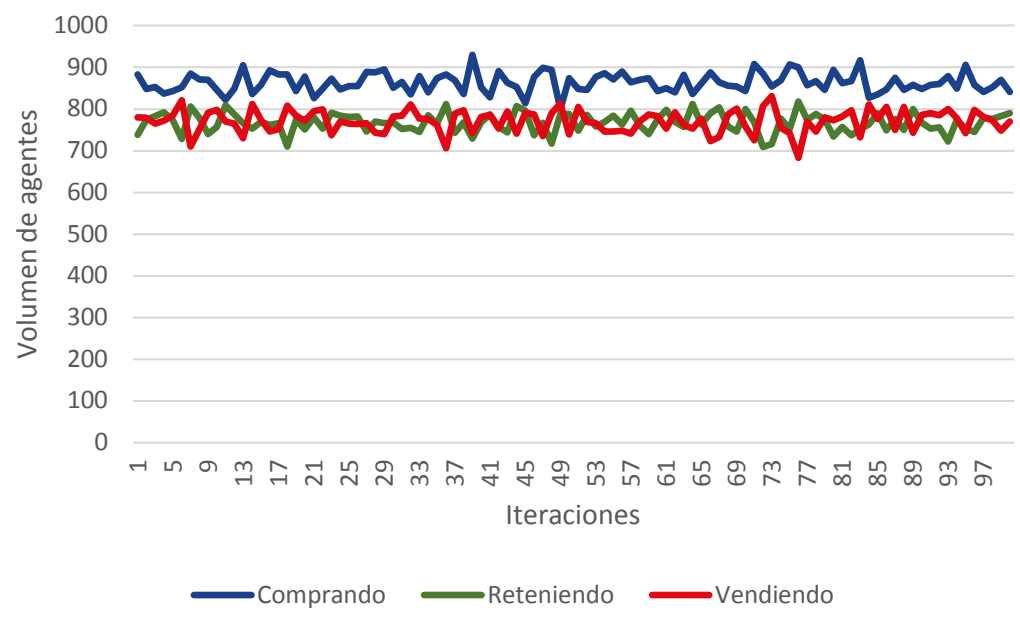

Fuente: Los autores

\section{Conclusiones}

- Al partir del modelo conductual propuesto por Fan en 2009 y el racional desarrollado en esta investigación, se obtienen los dos extremos de una discusión teórica: el primero busca modelar agentes que toman sus decisiones según la influencia de su vecindad, similar a la Hipótesis de Mercado Conductual propuesta por Shiller; el segundo obtiene la información de la dinámica de la Bolsa, simulada como un comportamiento lógico propio de la Hipótesis de Mercado Eficiente expuesta por Fama; en ambos casos, ninguna propuesta pareciese satisfacer el comportamiento de un mercado de valores en madurez. Lo anterior permite proponer para futuras investigaciones que los mercados simulados deben poseer características diversas, incluyendo diferentes tipos de agentes y reglas de evolución particulares, con las que se modelará la interacción entre conceptos contrarios como son las decisiones racionales y las conductuales.

- Existen metodologías para construir precios y evaluar si estos son similares a los reales, tal cual expone Milan en 2011; sin embargo, dichos modelos no logran captar toda la interacción entre los agentes de una Bolsa, por ende, se recomienda integrar la generación de precios a los modelos basados en agentes con el fin de evaluar cómo la caracterización de personas o inversores puede impactar de forma no lineal en la construcción de precios y, en consecuencia, en el desarrollo de una economía desde la perspectiva computacional. 
- Dentro de los mercados bursátiles artificiales existen dos grandes categorías: modelos generadores de precios o algoritmos de precios, los cuales tienen un enfoque aplicado y, por tanto, suelen ser más llamativos para la industria de inversión, ya que permiten analizar las rentabilidades futuras de sus activos financieros; $y$, por otra parte, los modelos conceptuales como aquellos desarrollados por Fan y Mozafari que facilitan el entendimiento de la conducta humana simulada y el contraste de nuevas teorías basadas en modelos matemáticos o físicos. Como conclusión general es recomendable trabajar con ambas categorías mediante el modelamiento basado en agentes, puesto que este invita a la investigación multidisciplinar, incluyendo psicología, sociología, econometría, finanzas, matemáticas, física y demás ciencias humanas o exactas, a la par que genera transferencia de conocimiento mediante el pronóstico de series reales.

\section{Referencias Bibliográficas}

- $\quad$ ASHBY, Ross. (1987). Sistemas y sus medidas de Información. Tendencias en la teoría general de sistemas. 3ra ed. Buenos aires: Alianza Editorial. 328 Pp.

- $\quad$ BACHELIER, Louis. (1900). Theorie de La Speculation. Theory of Speculation. (Tesis doctoral) Paris-Sorbonne University, Paris, Francia.

- BLÁSQUEZ, Benjamín. (2000). Bolsa y estadística bursátil, 1 ra ed. Madrid: Ediciones Díaz de Santos. 328 Pp.

- DUARTE, Juan Benjamín; GARCÉS, Laura Daniela y SIERRA, Katherine Julieth. (2016). Efecto manada en sectores económicos de las bolsas latinoamericanas: una visión pre y poscrisis subprime. En: Contaduría y Administración, 61 (2), 298323, Ciudad de México D.F.: Universidad Nacional Autónoma de México.

- DUARTE, Juan Benjamín y MASCAREÑAS, Juan Manuel (2014). ¿Han sido los mercados bursátiles eficientes informacionalmente? En: Apuntes del CENES, 33 (57), 117-146. Cali: Universidad Pedagógica y Tecnológica de Colombia.

- DUARTE, Juan Benjamín y MASCAREÑAS, Juan Manuel. (2014). Comprobación de la eficiencia débil en los principales mercados financieros latinoamericanos. En: Estudios Gerenciales, 30 (133), 365-375. Cali: Universidad ICESI.

- DUARTE, Juan Benjamín; MASCAREÑAS, Juan Manuel y SIERRA, Katherine Julieth. (2014). Testing the efficiency market hypothesis for the Colombian stock market. En: DYNA, 81 (1), 1-10. Bogotá: Universidad Nacional de Colombia.

- $\quad$ FAMA, Eugene. (Enero 1965). The Behavior of Stock-Market Prices. En Journal of Business, 38 (1), 34-105. Chicago: University of Chicago Press.

- $\quad$ FAMA, Eugene. (1970). Efficient capital markets: a review of theory and empirical work. En: The Journal of Finance. № 25 (2), 383-417. Hoboken (US): American Finance Association.

- $\quad$ FAN, Ying; SHANG.-Jun, Ying; WANG, Bing-Hong y WEI, Yi-Ming. (2009). The effect of investor psychology on the complexity of stock market: An analysis based on cellular automaton model. En: Computers \& Industrial Engineering, 56 (1), 63-69. Los Angeles (US): Elsevier.

- GIBSON, George Rutledge (1889). The stock exchanges of London, Paris, and New York : a comparison, 1 ra Ed. New York: Putman \& Sons. 155 Pp. 
- KEYNES, John Maynard. (1923). Some Aspects of Commodity Markets. En: Manchester Guardian Commercial: European Reconstruction Series. 12 (1), 255266. Manchester: John Russell Scott Editors.

- $\quad$ LEBARON, Blake; ARTHUR, Brian y PALMER, Richard (1999). Time series properties of an artificial stock market. En: Journal of Economic Dynamics and Control, 23 (9-10), 1487-1516. Boston (US): Elsevier.

- LEVY, Moshe; LEVY, Haim y SOLOMON, Sorin. (2000). The LLS microscopic simulation model. (Pp. 141-182). En: LEVY, Moshe et al. Microscopic Simulation of Financial Markets. San Diego (US): Academic Press.

- LO, Andrew. (2005). Reconciling Efficient Markets with Behavioral Finance: The Adaptive Markets Hypothesis. En: Journal of Investment Consulting, 7 (2), 21-44. Chicago US): IMCA Ed.

- LOVRIC, Milan. (2011). Behavioral Finance and Agent-Based Artificial Markets. (Tesis doctoral) Erasmus University Rotterdam, Rotterdam, Holanda.

- MANDELBROT, Benoit. (1972). Statistical methodology for nonperiodic cycles: from the covariance to R/S analysis. En: National Bureau of Economic Research. Annals of Economic and Social Measurement, 1 (3), 259-290. New York: National Bureau of Economic Research.

- MOZAFARI, Milad y ALIZADEH, Rahim (2013). A cellular learning automata model of investment behavior in the stock market. En: Neurocomputing, 122 (1), 470479. Ámsterdam: ELSEVIER.

- PETERS, Edgar (1994). Fractal Market Analysis: Applying Chaos Theory to Investment and Economics, 1 ra Ed. New York: John Wilei \& Sons. 324 Pp.

- QUEZADA, Ariel. (2007, April 17). Fractales y opinión pública: Una aplicación del exponente de Hurst al estudio de la dinámica de la identificación ideológica. (Tesis doctoral) Universitat de Barcelona. Barcelona, España.

- $\quad$ SHILLER, Robert. (2003). From efficient markets theory to behavioral finance. En: Journal of Economy Perspectives, 17 (1), 83-104. New Haven (US): Yale University.

- SHILLER, Robert. (2006). Tools for Financial Innovation: Neoclassical versus Behavioral Finance. En: Financial Review, 41 (1), 1-8. New Haven (US): Yale University.

- $\quad$ SIDNEY, Alexander. (1961). Price Movements in Speculative Markets: Trends or Random Walk? En: Industrial Management Review, 7, 7-26, Cambridge: University of Cambridge.

- $\quad$ SIERRA, Katherine; DUARTE, Juan Benjamín y MASCAREÑAS, Juan Manuel. (2013). Comprobación del comportamiento caótico en Bolsa de valores de Colombia. En: Estrategia Organizacional, 2 (1) 41-54. Bogotá: Universidad Nacional Abierta y a Distancia.

- TALERO, Leonardo Hernán. (2015). Comprobación de la teoría de finanzas conductuales en el mercado bursátil de Colombia mediante la simulación de mercados artificiales. (Tesis de pregrado), Universidad Industrial de Santander, Bucaramanga, Colombia.

- TALERO, Leonardo Hernán, DUARTE, Juan Benjamín y SIERRA, Katherine Julieth (9 de mayo de 2015). Evaluación de las metodologías para la construcción de mercados artificiales. Medellín, Antioquia, Colombia: IV Congreso internacional de finanzas: inversiones, Segundo encuentro de investigación en finanzas. doi:10.13140/RG.2.2.18717.10722

Para citar este artículo:
Talero, L.; Duarte, J.B. \& Sierra, K.J. (2017). Evaluación de metodologías para la construcción de mercados bursátiles artificiales. Teuken Bidikay Vol. 8 N¹1. Pp. 81-97 
Libre es el que es capaz todavía de elegir la defensa de su dignidad en un mundo donde, quieras o no, en algún momento tendrás que tomar partido entre los indignos y los indignados. No nací para obedecer. Nací para ejercer mi libertad de conciencia. No puedo aceptar la idea de que entre las personas o entre los países, haya conductores y conducidos. Desde la conquista española hemos sido entrenados por imperios sucesivos para la ignorancia mutua, para el divorcio y el odio mutuos. La especialidad latinoamericana es la guerra de vecinos.

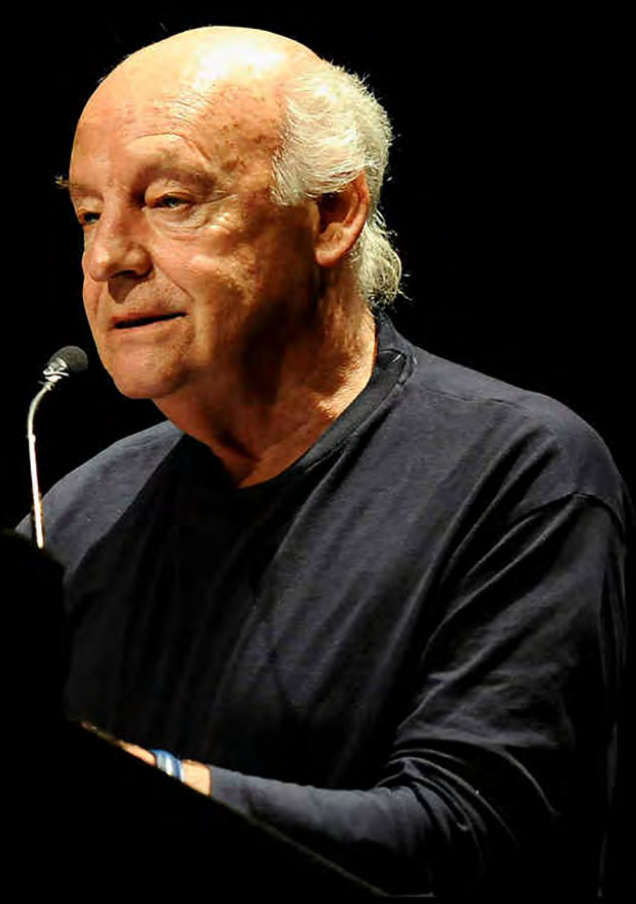

